\title{
Conductivity Mismatch and Voltage Dependence of Magnetoresistance in a Semiconductor Spin Injection and Detection Structure
}

\author{
Arunanshu M. Roy \\ Center for Integrated Systems \\ Stanford University \\ Stanford, California 94305 USA \\ Email: amroy@stanford.edu
}

\author{
Dmitri E. Nikonov \\ Technology Strategy \\ Intel Corporation \\ Santa Clara, California 95052 USA \\ Email: dmitri.e.nikonov@intel.com
}

\author{
Krishna Saraswat \\ Center for Integrated Systems \\ Stanford University \\ Stanford, California 94305 USA \\ Email: saraswat@cis.stanford.edu
}

\begin{abstract}
This work studies the voltage dependence of magnetoresistance in a semiconductor spin injection and detection structure. The voltage dependence of the spin dependent interface resistance at a ferromagnet semiconductor interface is investigated and spin diffusion models are used to evaluate the voltage dependence of magnetoresistance. We find that the voltage dependence of magnetoresistance is similar to that in magnetic tunnel junctions. Using our model for spin injection in a ferromagnet-oxide-semiconductor junction, the variation of magnetoresistance ratio with semiconductor doping and oxide thickness has been studied.
\end{abstract}

\section{INTRODUCTION}

Injection of spin polarized current into semiconductors has garnered great interest recently with applications to FETs and memory devices. Since the pioneering proposal of the spin transistor by Datta and Das [1], several experiments have confirmed the possibility of spin injection into semiconductors like GaAs and Si. Experiments on GaAs have been based on optical and electrical detection [2]-[4] while those on Si have utilized all-electrical effects such as non-local measurements [5] or the use of hot electron spin injectors [6]. Magnetoresistance (MR) devices based on spin injection and detection through semiconductors suffer from the problem of conductivity mismatch [7] between the ferromagnetic spin injector and the semiconductor layer. It has been shown that by using a suitable spin dependent interface resistance the problem of conductivity mismatch can be overcome [8]. This work studies in more detail the MR properties of a semiconductor layer sandwiched between ferromagnetic layers using spin dependent tunneling resistance to overcome the conductivity mismatch problem. In particular, we develop a numerical model to account for aspects important in practical spintronic devices: the voltage dependent interface resistance and magnetoresistance and the voltage and charge distribution in the depletion region of the semiconductor. An analysis of relevant semiconductor and oxide parameters is performed to design structures with an optimized MR ratio.

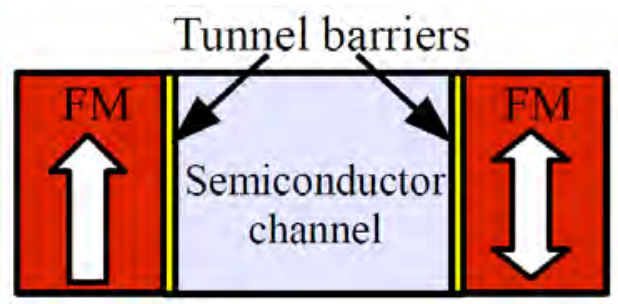

Fig. 1. Schematic diagram of semiconductor spin injection and detection structure. When a voltage is applied to the structure, one junction is forward biased and the other is reverse biased

\section{Methodology}

A typical semiconductor spin injection and detection structure consisting of a semiconductor layer sandwiched between two ferromagnetic layers is shown in Fig. 1.

Tunnel barriers are used as a spin dependent interface resistance. Spin transport simulations are based on the 1D spin diffusion model as proposed by Fert and Jaffres [9]. The spin dependent interface resistance is one of the most crucial parameters in spin diffusion simulations as it controls the splitting of electrochemical potential at an interface. The following sections briefly describe the mathematical equations used to model spin diffusion and interface I-V characteristics.

\section{A. Spin diffusion model}

Using the approach of Valet and Fert [10], a macroscopic model for spin diffusion in a multilayer stack of materials is characterised by spin diffusion equations. A short review of the mathematical model used is provided here.

$$
\begin{gathered}
\frac{\partial^{2} \Delta \mu}{\partial z^{2}}=\frac{\Delta \mu}{l_{s f}^{2}} \\
\frac{\partial^{2}\left(\sigma_{+} \mu_{+}+\sigma_{-} \mu_{-}\right)}{\partial z^{2}}=0
\end{gathered}
$$

The spin diffusion is described in terms of the difference in electrochemical potentials for electrons with spin $+\frac{1}{2}$ and spin $-\frac{1}{2}$ by (1). The parameter $l_{s f}$ called the spin flip 
length is characteristic of the material. Current continuity is ensured through (2). The subscripts here refer to electrons with spin $+\frac{1}{2}$ and spin $-\frac{1}{2}$. Thus $\mu_{+}$and $\mu_{-}$represent the electrochemical potentials and the conductivity is represented by $\sigma_{+}$and $\sigma_{-}$. The conductivity itself is modeled in terms of material parameter $\beta$ using (3).

$$
\rho_{\uparrow(\downarrow)}=\frac{1}{\sigma_{\uparrow(\downarrow)}}=2 \rho^{*}[1-(+) \beta]
$$

The resistivity $\rho_{\uparrow}$ and $\rho_{\downarrow}$ refer to the resistivity for the majority and minority electrons respectively in the material. This notation $\uparrow(\downarrow)$ is also used to label other variables majority(minority) electrons. A general solution to this set of equations can be written as follows. Note that K1, K2, K3 are constants for every layer and we need to choose appropriate values for these constant that satisfy boundary conditions.

$$
\begin{aligned}
\mu_{\uparrow}= & \left(1-\beta^{2}\right) q \rho_{f}^{*} J z+K_{1}+ \\
& (1+\beta)\left[K_{2} \exp \left(\frac{z}{l_{s f}}\right)+K_{3} \exp \left(\frac{-z}{l_{s f}}\right)\right] \\
\mu_{\downarrow}= & \left(1-\beta^{2}\right) q \rho_{f}^{*} J z+K_{1}- \\
& (1-\beta)\left[K_{2} \exp \left(\frac{z}{l_{s f}}\right)+K_{3} \exp \left(\frac{-z}{l_{s f}}\right)\right] \\
J_{\uparrow}= & (1-\beta) \frac{J}{2}+ \\
& \frac{1}{2 q \rho_{f}^{*} l_{s f}}\left[K_{2} \exp \left(\frac{z}{l_{s f}}\right)-K_{3} \exp \left(\frac{-z}{l_{s f}}\right)\right] \\
J_{\downarrow}= & (1+\beta) \frac{J}{2}- \\
& \frac{1}{2 q \rho_{f}^{*} l_{s f}}\left[K_{2} \exp \left(\frac{z}{l_{s f}}\right)-K_{3} \exp \left(\frac{-z}{l_{s f}}\right)\right]
\end{aligned}
$$

For non-magnetic material layers, the same equations apply but $\beta=0$. Under the assumption that current continuity is maintained and there is no spin flipping at an interface, we have the following boundary conditions at interface $z=z_{0}$ in terms of an interface resistance area product parameter $r_{b}^{*}$ and spin selectivity parameter $\gamma$.

$$
\begin{aligned}
\Delta \mu_{\uparrow}\left(z_{0}\right) & =2 q r_{b}^{*}(1-\gamma) J_{\uparrow} \\
\Delta \mu_{\downarrow}\left(z_{0}\right) & =2 q r_{b}^{*}(1+\gamma) J_{\downarrow}
\end{aligned}
$$

These equations have been formulated in the transfer matrix formalism. This makes it suitable for studying multilayer devices with interfaces and also semiconductors with graded doping where the spin diffusion properties vary with semiconductor doping density.

\section{B. Model for the interface I-V characteristics}

As shown in Fig. 2 a simple model for a ferromagnet band structure consists of a majority and a minority band which are split due to exchange interaction. The current for each band is calculated by treating the majority and minority bands independently. Using the Tsu-Esaki model, the current through the interface for one band is given by (10).

$$
J_{t o t}=\frac{2 \pi m_{e f f} q}{h^{3}} \int_{E_{\min }}^{E_{\max }} \eta\left(\varepsilon_{z}\right) N_{t o t}\left(\varepsilon_{z}\right) d \varepsilon_{z}
$$

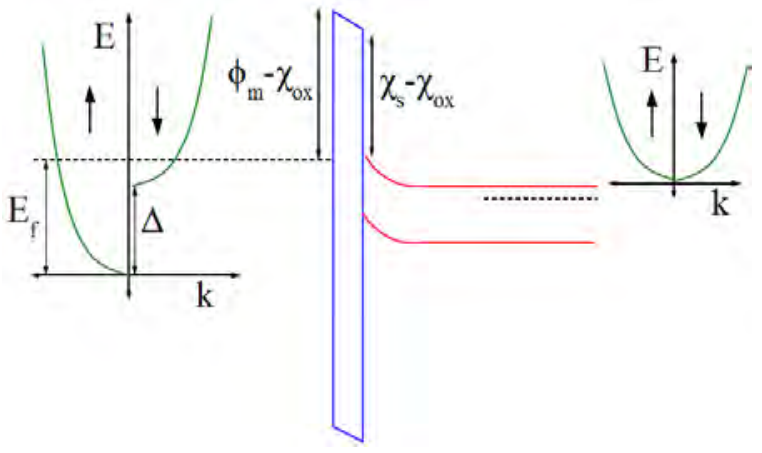

Fig. 2. The exchange splitting between the electrons in the majority and minority bands of the ferromagnet gives rise to a spin dependent contact resistance

where $m_{e f f}$ is a density of states effective mass, $h$ is Planck's constant, $\eta$ is the electron tunneling probability and $N_{t o t}$ is a supply function which depends on the Fermi energy in metal and semiconductor.

With an assumption of parabolic bands, Fermi Dirac statistics for electrons in the conduction bands and when the transmission probability is assumed to be independent of the parallel wave vector at the interface, the supply function $\mathrm{N}_{t o t}$ is given by (11).

$$
N_{t o t}=\left(k_{b} T\right) \ln \left\{\frac{1+\exp \left(\frac{\varepsilon_{f m}-\varepsilon_{z}}{k_{b} T}\right)}{1+\exp \left(\frac{\varepsilon_{f s}-\varepsilon_{z}}{k_{b} T}\right)}\right\}
$$

where $\varepsilon_{f m}$ is the Fermi energy in the metal, $\varepsilon_{f s}$ is the Fermi energy in the semiconductor, $k_{b}$ is the Boltzmann constant and $T$ is the absolute temperature.

To calculate the tunneling probability for a given applied voltage, the depletion region and band structure in the semiconductor region is calculated by solving the 1D Poisson equation and using equilibrium charge concentrations consistent with the band bending. The value of the tunneling transmission probability is then calculated using the transfer matrix formalism for tunneling problems. Fig. 3 shows the calculated majority and minority currents for spin injection into n-type $\mathrm{Si}$ with an $\mathrm{Al}_{2} \mathrm{O}_{3}$ tunnel barrier. The interface resistance depends upon the tunnel barrier thickness and the semiconductor Schottky barrier. The spin dependence of the tunneling currents is mainly due to the lack of minority states near the Fermi energy in the ferromagnet.

\section{Voltage Dependence of Magnetoresistance RATIO}

Since previous simulations have used a constant spin dependent interface resistance, they fail to capture the effect of the voltage dependence of a tunnel barrier interface resistance and also the fact that I-V characteristics of a tunnel barrier are not necessarily symmetric for positive and negative voltages. The spin dependent interface resistance is modeled as in [9] 


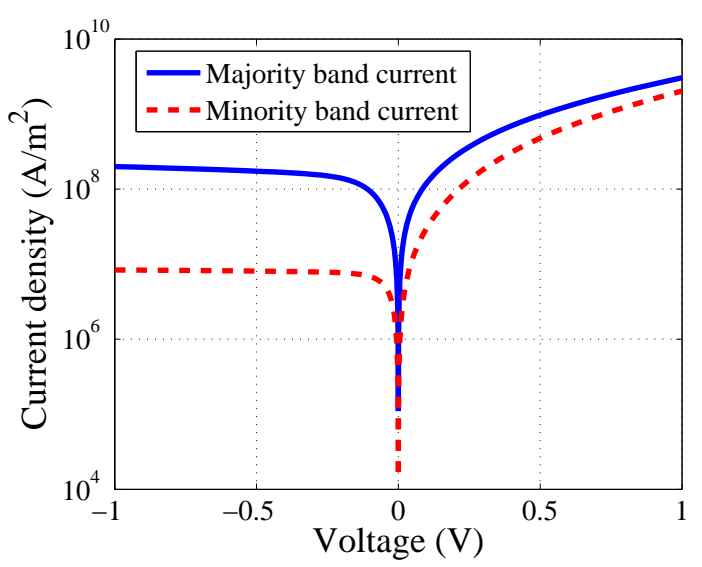

Fig. 3. Simulated currents for majority and minority bands. The structure used is metal $\left(\phi_{m}=4 \mathrm{eV}, \mathrm{E}_{f}=2.2 \mathrm{eV},=2.2 \mathrm{eV}\right) \mathrm{Al}_{2} \mathrm{O}_{3}$ (8 Angstrom) $\mathrm{Si}$ (N- type doping $10^{16} \mathrm{~cm}^{-3}$ )

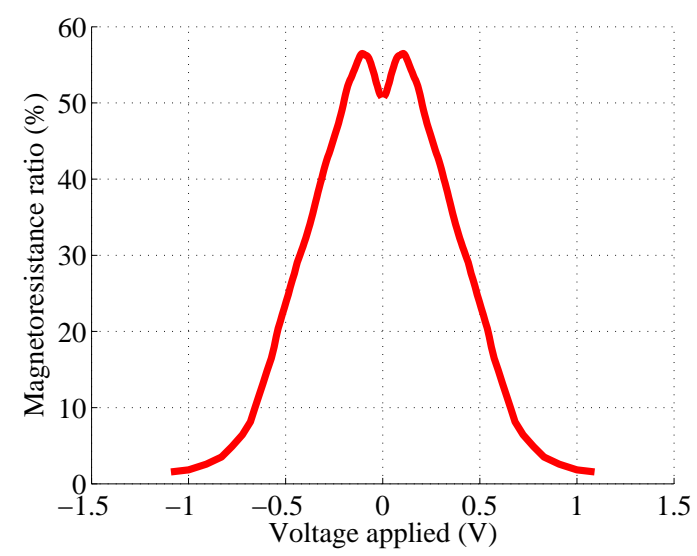

Fig. 4. Voltage dependence of magnetoresistance for structure in Fig. 1. The interface resistance and spin selectivity is calculated using the current voltage characteristics shown in Fig. 3. Length of the semiconductor channel is 200 $\mathrm{nm}$.

in terms of constant parameters $r_{b}$ (interface resistance area product) and $\gamma$ (spin selectivity). Thus the interface resistance area product is $2 r_{b}(1-\gamma)$ for the majority electrons and $2 r_{b}(1+\gamma)$ for the minority electrons. Here however the computed I-V characteristics for majority and minority bands are used self consistently with the majority and minority electrochemical potentials in the spin diffusion simulations to compute $r_{b}$ and $\gamma$ (a voltage and spin dependent interface resistance for each interface). As shown in Fig. 4, the MR of these structures is maximal when the potential drop across the structure nears zero volts.

This is similar to the experimentally observed characteristics of magnetic tunnel junctions [11]. Figs. 5 and 6 show that both interface resistance area product and the spin selectivity of the forward and reverse biased contacts are very different and they vary substantially with voltage applied to the structure.

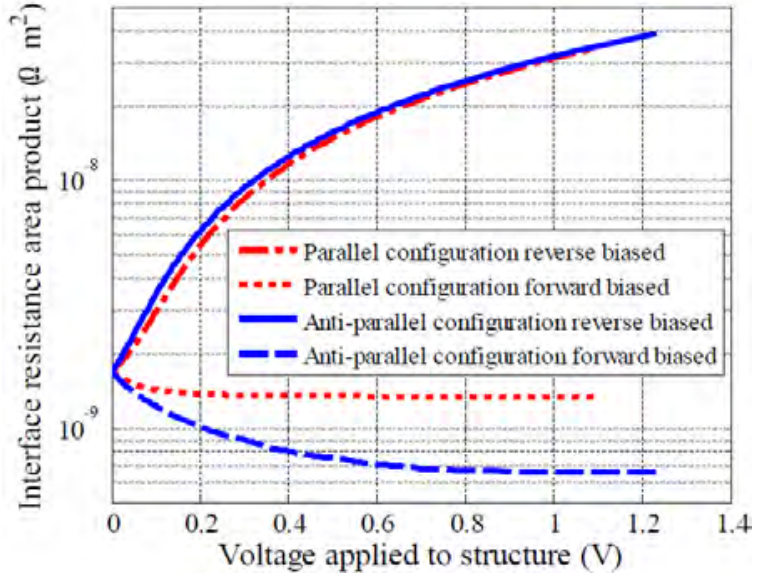

Fig. 5. Voltage dependence of interface resistance area product for simulations in Fig. 3. Reverse biased junctions show a substantial increase in interface resistance area product with applied voltage

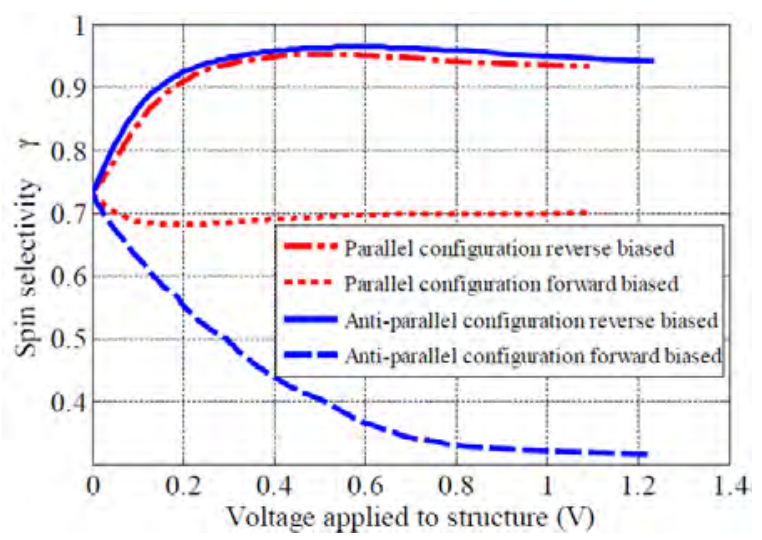

Fig. 6. Voltage dependence of spin selectivity of the interfaces shows that the forward and reverse biased junction have very different spin selectivity which varies significantly with the applied voltage

\section{EFFECT OF MATERIAL PARAMETERS ON MAGNETORESISTANCE}

Using the MR at zero volts as a metric, we study the effect of parameters such as oxide thickness, semiconductor doping and ferromagnet band structure. The spin selectivity of tunnel barrier is affected most strongly by the ferromagnet band structure. As we approach a half metallic ferromagnet with the minority conduction band minimum at or above the Fermi level, the spin selectivity improves greatly since the Fermi distribution ensures that minority currents are low. In this model the oxide thickness does not affect the spin selectivity but needs to be tuned for the appropriate contact resistance area product. For crystalline $\mathrm{MgO}$ this model is inadequate and it is expected that spin selectivity will increase with oxide thickness. The semiconductor doping determines the required interface contact resistance area product which itself is determined by the metal work-function, the oxide thickness and the semiconductor doping. We study the effect of these parameters on MR. In Figs. 7,8 and 9 the variation of MR at zero volts with oxide thickness, semiconductor doping and 


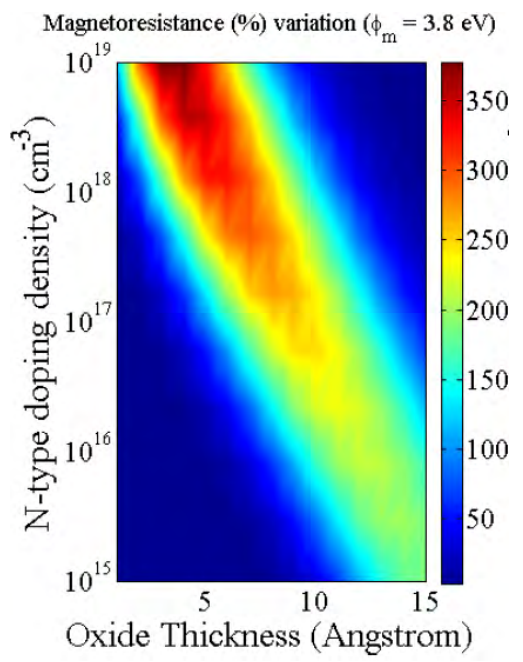

Fig. 7. Ferromagnet work function $=3.8 \mathrm{eV}$. A low work function gives higher MR due to a lower resistance area product and higher spin selectivity at the interfaces

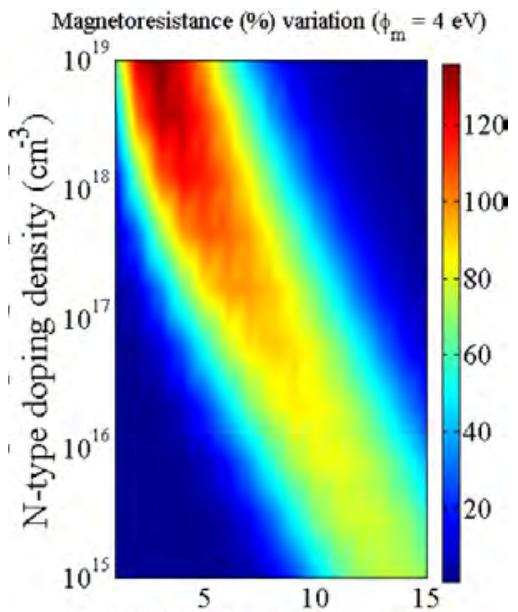

Oxide Thickness (Angstrom)

Fig. 8. Ferromagnet work function $=4 \mathrm{eV}$. Increasing the resistance area product reduces the MR. Higher doping values need lower oxide thickness

metal work-function is shown. The tunnel barrier is $\mathrm{Al}_{2} \mathrm{O}_{3}$, the semiconductor is n-type $\mathrm{Si}$ and the ferromagnet is nearly half metallic with the bottom of the minority conduction band coinciding with the Fermi energy. A lower metal workfunction increases the MR significantly and for a given workfunction the plots show the optimal values of doping and oxide thickness.

\section{CONCLUSION}

We demonstrate that the voltage dependence of interface resistance captured by this numerical model, significantly affect the resulting MR. The voltage dependence of MR is moderate and comparable to that in magnetic tunnel junctions. Our models and implementation are both general and efficient. They can be used to study the effect of material parameters as well as doping effects, bringing us closer to designing a working spinFET.

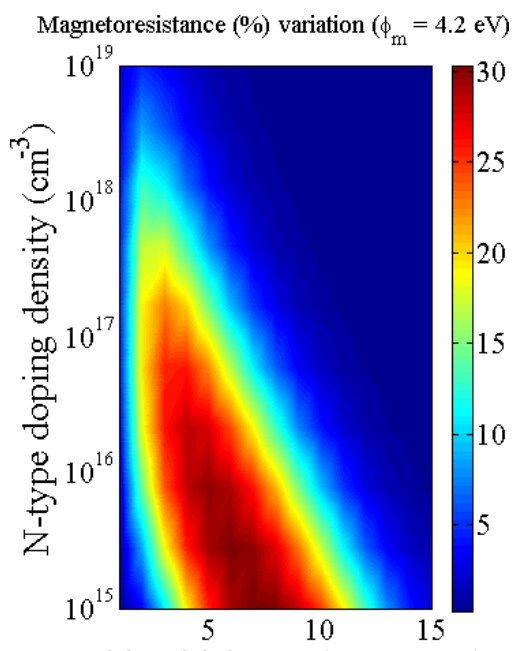

Oxide Thickness (Angstrom)

Fig. 9. For ferromagnet work function $=4.2 \mathrm{eV}$, the interface resistance area products are too high and the maximum MR is close to only $30 \%$

\section{ACKNOWLEDGMENT}

The authors are grateful to Intel Corp. for providing support for this work and wish to acknowledge Prof. Shan Wang, Dr. Tejas Krishnamohan, J. Jason Lin and Donkoun Lee for helpful discussions.

\section{REFERENCES}

[1] S. Datta and B. Das, "Electronic analog of the electro-optic modulator," Appl. Phys. Lett., vol. 56, p. 665, Feb. 1990.

[2] R. Fiederling et al., "Injection and detection of a spin-polarized current in a light-emitting diode," Nature, vol. 402, pp. 787-790, Dec. 1999.

[3] X. Lou et al., "Electrical detection of spin transport in lateral ferromagnet semiconductor devices," Nature Physics, vol. 3, pp. 197-202, 2007.

[4] H. J. Zhu, M. Ramsteiner, H. Kostial, M. Wassermeier, H.-P. Schönherr, and K. H. Ploog, "Room-temperature spin injection from fe into gaas," Phys. Rev. Lett., vol. 87, no. 1, p. 016601, Jun 2001.

[5] O. M. J. vant́ Erve et al., "Electrical injection and detection of spinpolarized carriers in silicon in a lateral transport geometry," Appl. Phys. Lett., vol. 91, p. 212109, Nov. 2007.

[6] I. Appelbaum, B. Huang, and D. J. Monsma, "Electronic measurement and control of spin transport in silicon," Nature, vol. 447, pp. 295-298, May 2007.

[7] G. Schmidt, D. Ferrand, L. W. Molenkamp, A. T. Filip, and B. J. van Wees, "Fundamental obstacle for electrical spin injection from a ferromagnetic metal into a diffusive semiconductor," Phys. Rev. B, vol. 62, no. 8, pp. R4790-R4793, Aug 2000.

[8] E. I. Rashba, "Theory of electrical spin injection: Tunnel contacts as a solution of the conductivity mismatch problem," Phys. Rev. B, vol. 62 no. 24, pp. R16267-R16270, Dec 2000.

[9] A. Fert and H. Jaffrès, "Conditions for efficient spin injection from a ferromagnetic metal into a semiconductor," Phys. Rev. B, vol. 64, no. 18, p. 184420 , Oct 2001

[10] T. Valet and A. Fert, "Theory of the perpendicular magnetoresistance in magnetic multilayers," Phys. Rev. B, vol. 48, no. 10, pp. 7099-7113, Sep 1993.

[11] M. Hosomi et al., "A novel nonvolatile memory with spin torque transfer magnetization switching: spin-ram," in IEDM Technical Digest, 2005 , pp. 459-462. 